Supporting Information

\title{
Observation of the Kondo Effect in Multilayer Single-Crystalline VTe2 Nanoplates
}

Hongtao Liu, ${ }^{\dagger, \#}$ Yunzhou Xue, ${ }^{\S, \# ~ J i n-A n ~ S h i, ~}{ }^{\perp}$ Roger A. Guzman, ${ }^{\perp}$ Panpan Zhang, ${ }^{\dagger}$ Zhang Zhou, ${ }^{\dagger}$ Yangu He, ${ }^{\dagger}, \|$ Ce Bian, ${ }^{\dagger}$ Liangmei Wu, ${ }^{\dagger}$ Ruisong Ma, ${ }^{\dagger}$ Jiancui Chen, ${ }^{\dagger}$ Jiahao Yan, ${ }^{\dagger}$ Haitao Yang, ${ }^{\dagger}$ Cheng-Min Shen, ${ }^{\dagger}, \dot{+}$ Wu Zhou, ${ }^{\perp}$ Lihong Bao, ${ }^{*,+,+}$ Hong-Jun Gao ${ }^{\dagger}$

'Institute of Physics \& University of Chinese Academy of Sciences, Chinese Academy of Sciences, Beijing, 100190, P. R. China

${ }^{\S}$ College of Chemistry and Environmental Engineering, Shenzhen University, Shenzhen, 518060, P. R. China

${ }^{\perp}$ School of Physical Sciences and CAS Key Laboratory of Vacuum Physics, University of Chinese Academy of Sciences, Beijing 100049, China

Fongshan Lake Materials Laboratory, Dongguan, Guangdong, 523808, P. R. China

"Department of Physics, Applied Physics, and Astronomy, Rensselaer Polytechnic Institute, 110 Eighth Street, Troy, NY 12180, USA

*Corresponding author: E-mail:lhbao@iphy.ac.cn

"These authors contributed equally

This PDF file includes:

Figure S1. Sublimed-salt-assisted atmospheric pressure CVD growth of $\mathrm{VTe}_{2}$ on mica substrate

Figure S2. Optical images of the $\mathrm{VTe}_{2}$ nanoplates

Figure S3. Optical images of the as-grown $\mathrm{VTe}_{2}$ nanoplates with different thickness, and the $\mathrm{VTe}_{2}$ nanoplates stored in air for $\sim 2$ and $\sim 6$ months

Figure S4. SEM of an as-grown $\mathrm{VTe}_{2}$ nanoplate

Figure S5. SAED patterns of the as-grown $\mathrm{VTe}_{2}$ nanoplate collected from marked regions

Figure S6. TEM of an as-grown semi-round $\mathrm{VTe}_{2}$ nanoplate

Figure S7. Atomic-resolution of STEM image of the $\mathrm{VTe}_{2}$ nanoplate

Figure S8. Low temperature and high temperature electrical transport of the $\mathrm{VTe}_{2}$ nanoplate

Figure S9. Subtraction of quadratic ordinary contribution by fitting high field MR

Figure S10. Comparison of device performance between the as-grown and stored half a year in a glove box filled with $\mathrm{Ar}$

Figure S11. Low-temperature transport properties of another $\mathrm{VTe}_{2}$ nanoplate

Figure S12. Variation of Hall resistance, Hall coefficient, Hall concentration and Hall mobility with temperatures

Figure S13. Low-temperature transport properties of an ultrathin $\mathrm{VTe}_{2}$ nanoplate

Figure S14. High resolution XPS spectra of the as-grown, degraded and completely oxidized $\mathrm{VTe}_{2}$ nanoplates 
Figure S15. Structure model and DFT simulations of $\mathrm{VTe}_{2}$ with and without defects

Figure S16. Magnetization measurements of the as-grown $\mathrm{VTe}_{2}$ nanoplates

a


Figure S1. Sublimed-salt-assisted atmospheric-pressure CVD growth of $\mathrm{VTe}_{2}$ on mica substrate. (a) Schematic of the growth process by using $\mathrm{V}_{2} \mathrm{O}_{5} / \mathrm{NH}_{3} \mathrm{Cl}$ and Te as the precursors. (b) Average $\mathrm{VTe}_{2}$ nanoplate size plotted as a function of Te temperature with the temperature of $\mathrm{V}$ precursor (growth temperature) maintaining at $750{ }^{\circ} \mathrm{C}$. (c) Average thickness of the $\mathrm{VTe}_{2}$ nanoplates plotted as a function of growth temperature. 
a

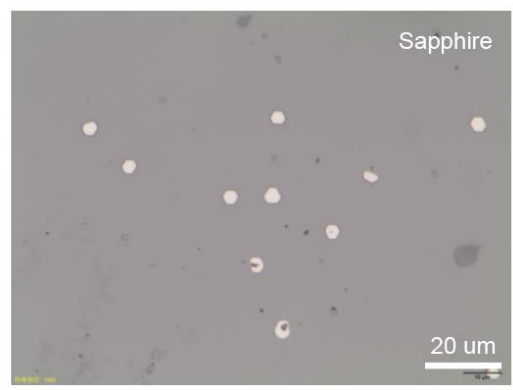

C

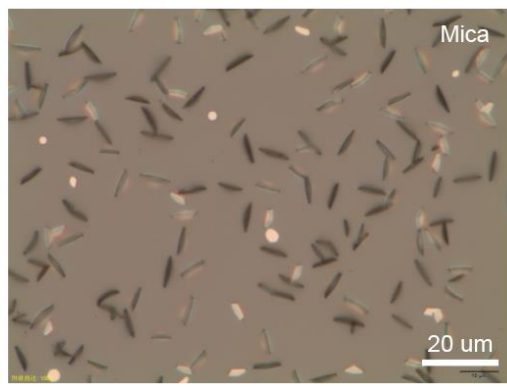

e





d

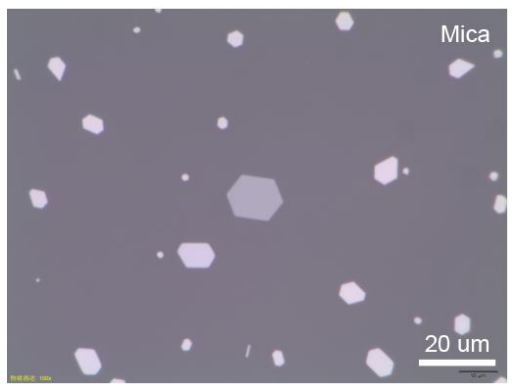

f

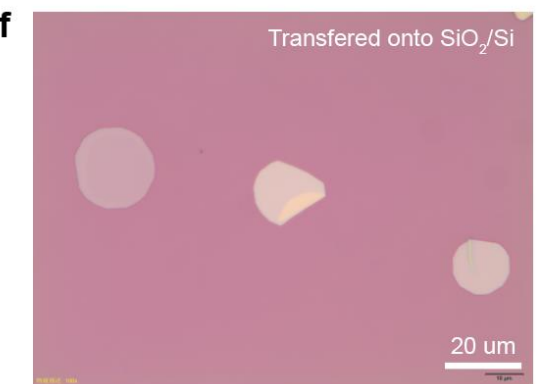

Figure S2. Optical images of the $\mathrm{VTe}_{2}$ nanoplates. $(\mathrm{a}, \mathrm{b})$ Optical images of the $\mathrm{VTe}_{2}$ nanoplates directly grown on sapphire substrate. A hexagonal $\mathrm{VTe}_{2}$ nanoplate segregates from Te droplet in (b). (c,d) Optical images of the $\mathrm{VTe}_{2}$ nanoplates directly grown on mica. Black lines in (c) are the $\mathrm{VTe}_{2}$ nanoplates vertically grown on the substrate. (e) The $\mathrm{VTe}_{2}$ nanoplates directly grown on $\mathrm{SiO}_{2} / \mathrm{Si}$ substrate. (f) The $\mathrm{VTe}_{2}$ nanoplates grown on mica substrate transferred on $\mathrm{SiO}_{2} / \mathrm{Si}$ substrate. 

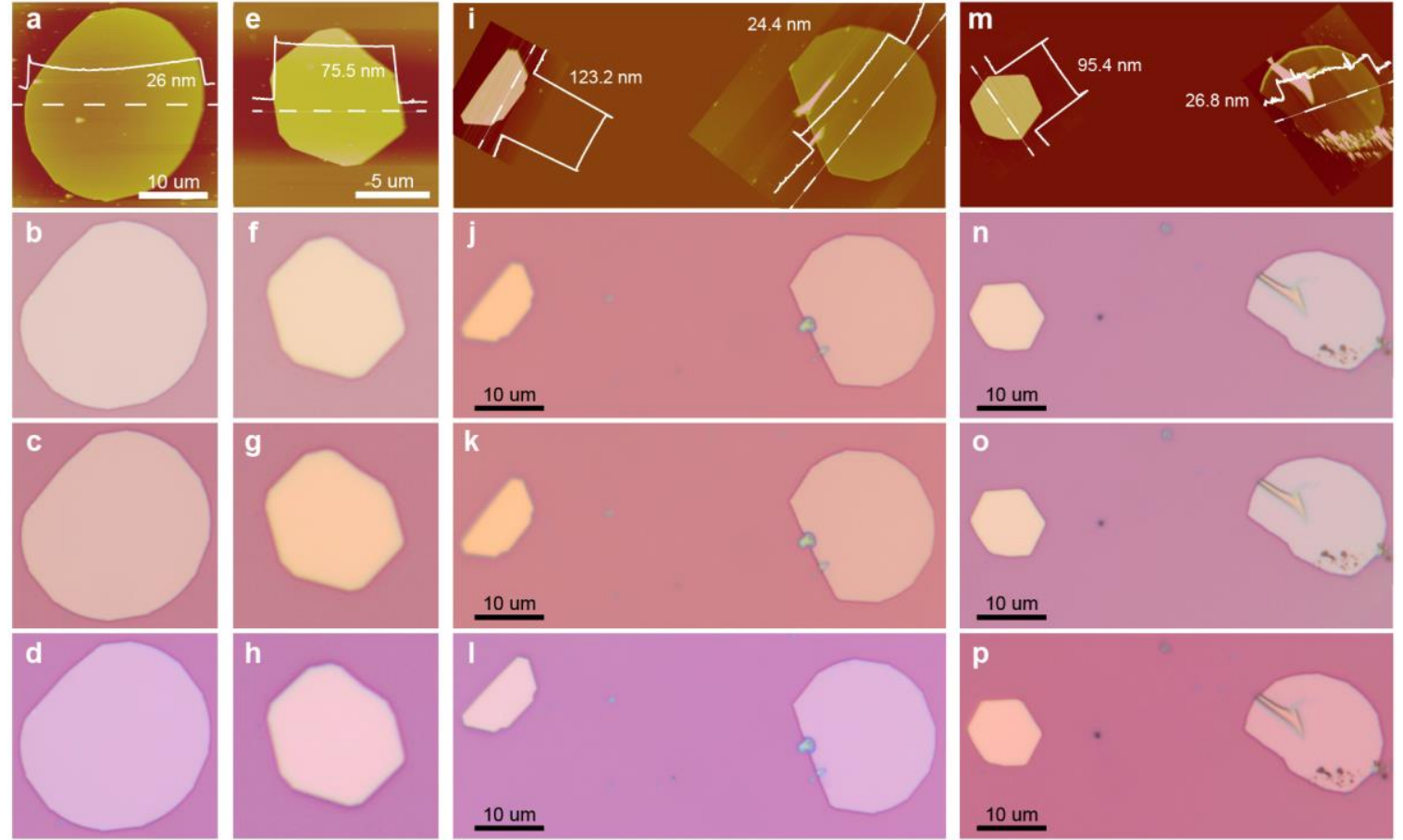

Figure S3. Optical images of the as-grown $\mathrm{VTe}_{2}$ nanoplates with different thickness, and the $\mathrm{VTe}_{2}$ nanoplates stored in air for $\sim 2$ and $\sim 6$ months. $(\mathrm{a}, \mathrm{e}, \mathrm{i}, \mathrm{m}) \mathrm{AFM}$ images of the $\mathrm{VTe}_{2}$ nanoplates with different shape and thickness. Optical images of the as-grown $\mathrm{VTe}_{2}$ nanoplates $(b, f, j, n)$, and the nanoplates exposed in air for about 2 months $(\mathrm{c}, \mathrm{g}, \mathrm{k}, \mathrm{o})$ and about 6 months $(\mathrm{d}, \mathrm{h}, \mathrm{l}, \mathrm{p})$, respectively.
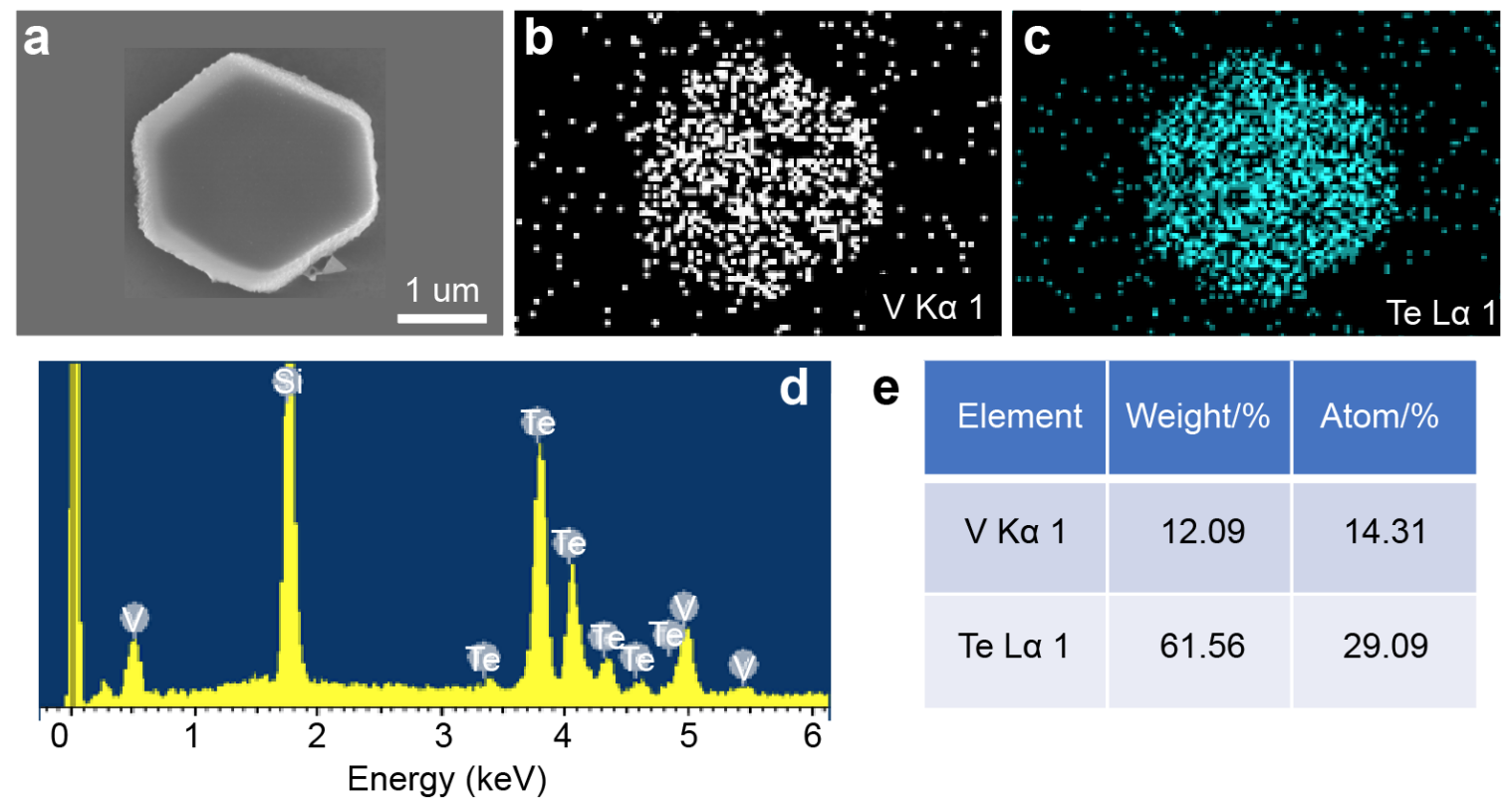

\begin{tabular}{|c|r|r|}
\hline Element & Weight/\% & Atom/\% \\
\hline V Ka 1 & 12.09 & 14.31 \\
\hline Te La 1 & 61.56 & 29.09 \\
\hline
\end{tabular}

Figure S4. SEM of an as-grown $\mathrm{VTe}_{2}$ nanoplate. (a-c) SEM image of a $\mathrm{VTe}_{2}$ nanoplate and the corresponding EDS mapping images of (b) V and (c) Te for the nanoplate. (d) EDS spectrum of the $\mathrm{VTe}_{2}$ nanoplate on $\mathrm{SiO}_{2} / \mathrm{Si}$ substrate. (e) Table of V/Te weight and atomic ratios obtained by EDS. 

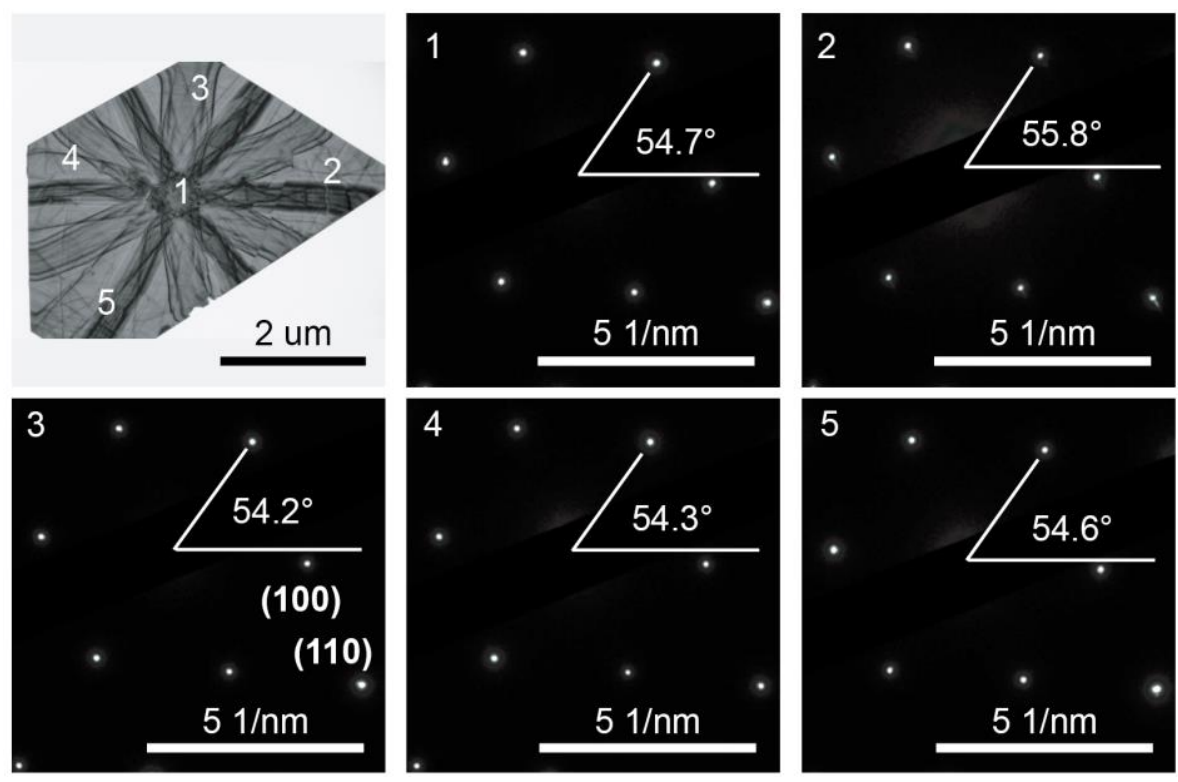

Figure S5. SAED patterns of the as-grown $\mathrm{VTe}_{2}$ nanoplate collected from marked regions.
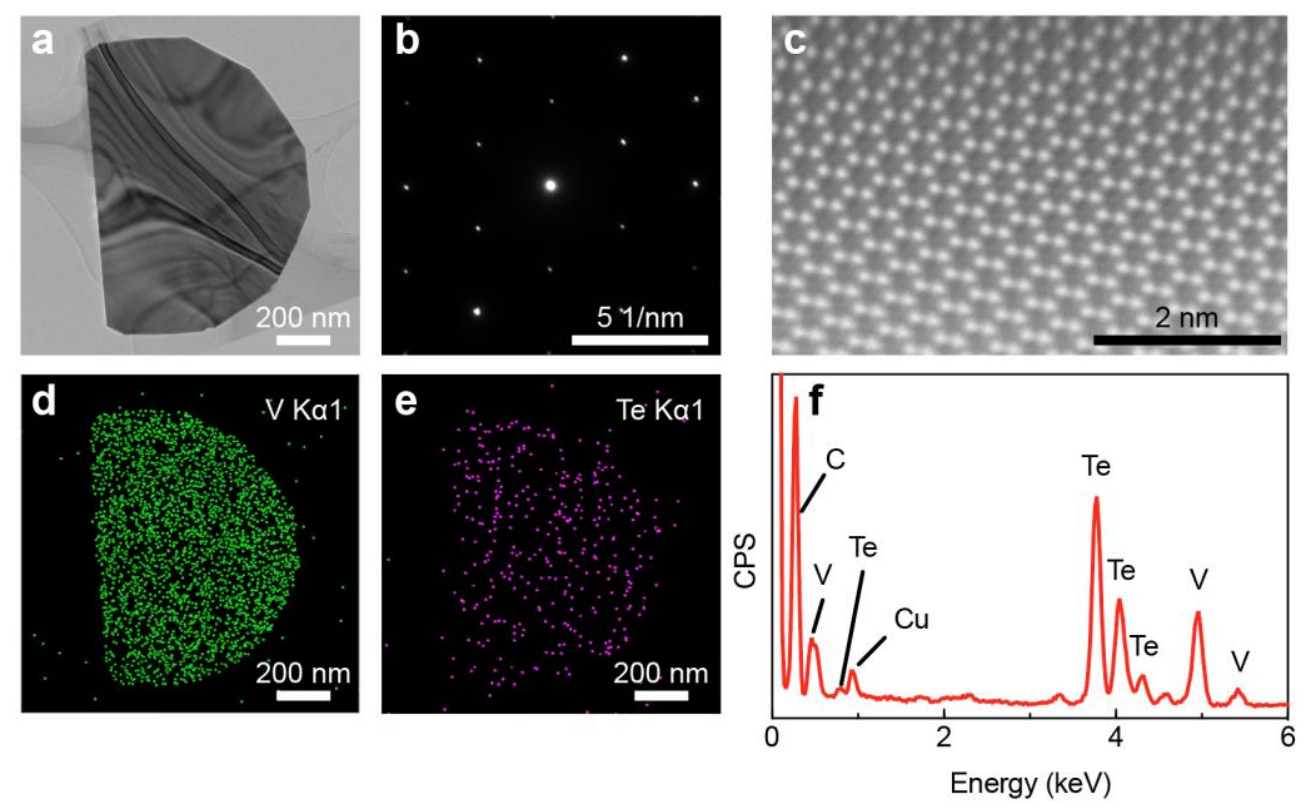

Figure S6. TEM of an as-grown semi-round $\mathrm{VTe}_{2}$ nanoplate. (a) Low magnification TEM image of a $\mathrm{VTe}_{2}$ nanoplate. (b) SAED pattern of the nanoplate. (c) Atomic-resolution HAADF-STEM image of the $\mathrm{VTe}_{2}$ nanoplate (filtered). EDS mapping images of (d) $\mathrm{V}$ and (e) Te of the nanoplate in (a). (f) EDS spectrum of the $\mathrm{VTe}_{2}$ nanoplate on $\mathrm{Cu}$ TEM grid showing a Te/V atomic ratio Te/V close to 2 . HAADFSTEM image in (c) was captured by Titan Cubed Themis G2 300, FEI, acceleration voltage of $300 \mathrm{kV}$. 

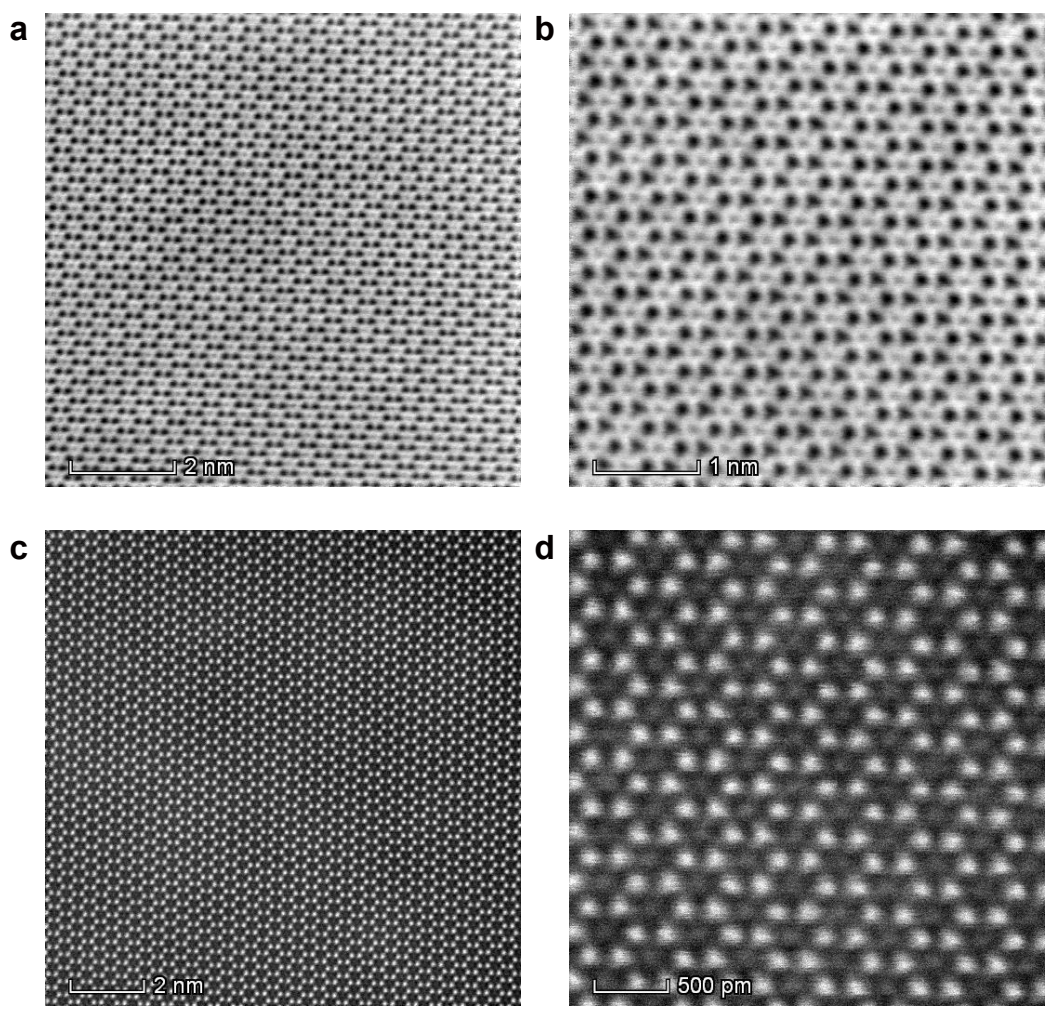

Figure S7. Atomic-resolution of STEM image of the $\mathrm{VTe}_{2}$ nanoplate. $(\mathrm{a}, \mathrm{b})$ Bright field STEM image of the $\mathrm{VTe}_{2}$ nanoplate with drift corrected frame integration (DCFI) of the $\mathrm{VTe}_{2}$ nanoplate. (c,d) Dark field STEM image of the $\mathrm{VTe}_{2}$ nanoplate with drift corrected frame integration (DCFI) of the $\mathrm{VTe}_{2}$ nanoplate. HAADF-STEM images were captured by Titan Cubed Themis G2 300, FEI, acceleration voltage of $300 \mathrm{kV}$.

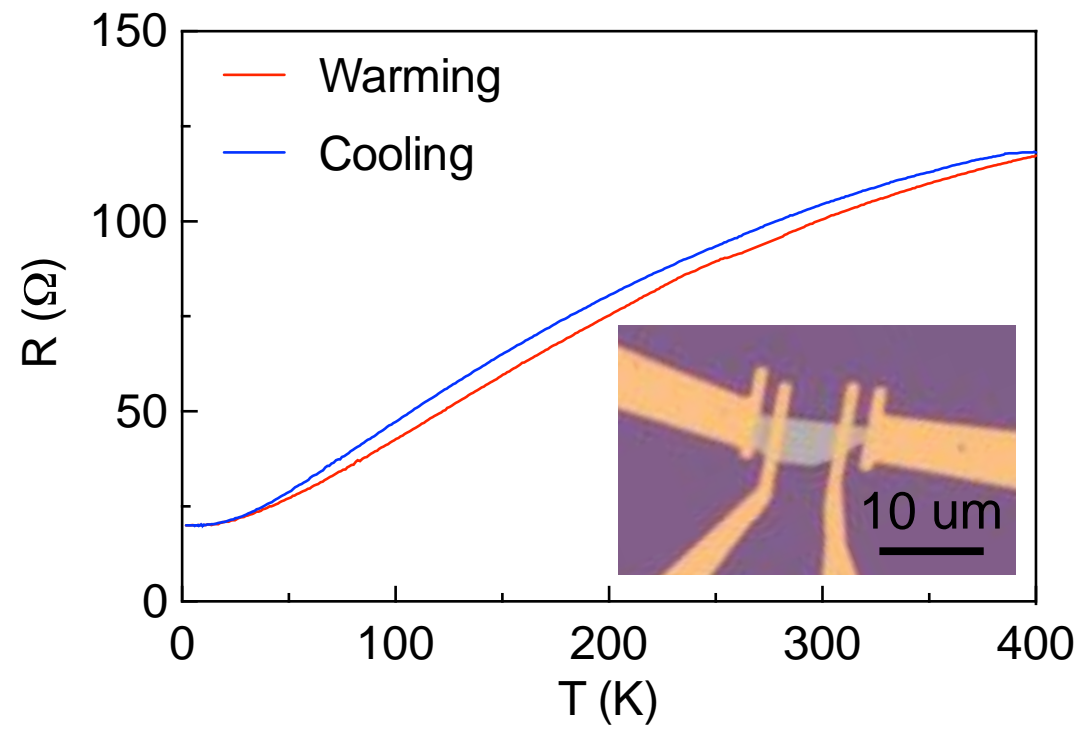

Figure S8. Low temperature and high temperature electrical transport of the $\mathrm{VTe}_{2}$ nanoplate with the optical image of the device showed in the inset. No obvious anomaly is observed in the temperature range from $400 \mathrm{~K}$ down to $1.9 \mathrm{~K}$. 

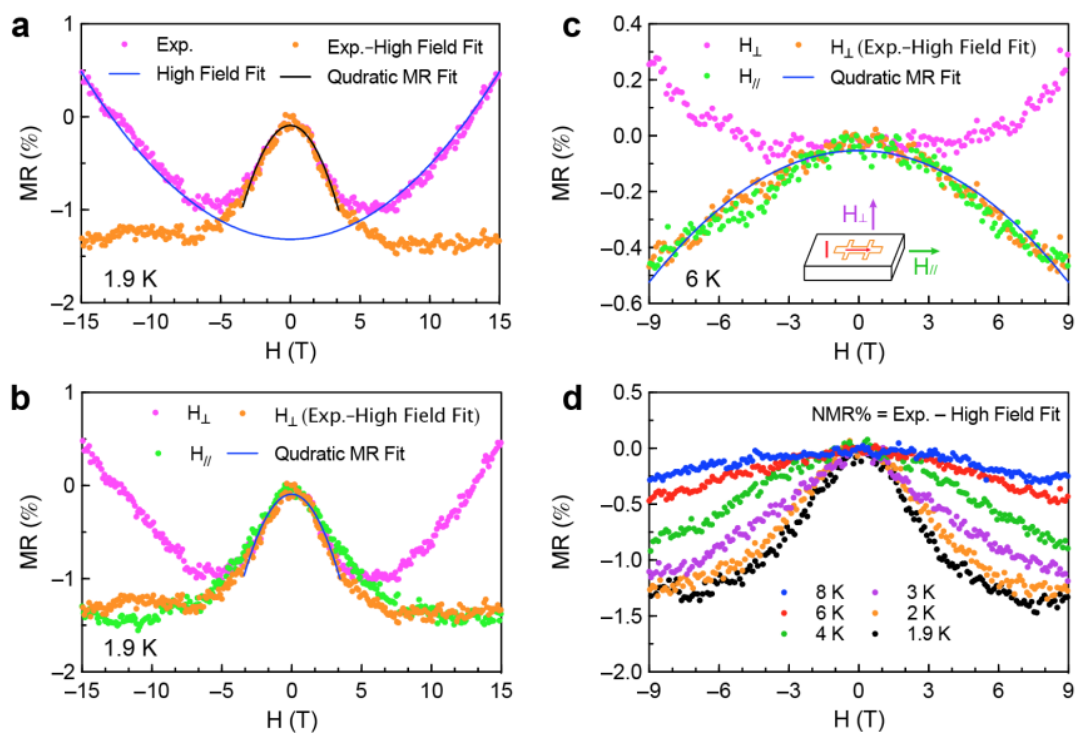

Figure S9. Subtraction of quadratic ordinary contribution by fitting high field MR. (a) Transverse MR, high field fit, low field fit, and the MR with quadratic ordinary MR subtracted by fitting the high field $\mathrm{MR}$ at $1.9 \mathrm{~K}$. (b,c) Comparison of the transverse MR and longitudinal MR at $1.9 \mathrm{~K}$ and $6 \mathrm{~K}$ respectively. (d) Transverse NMR at different temperatures.
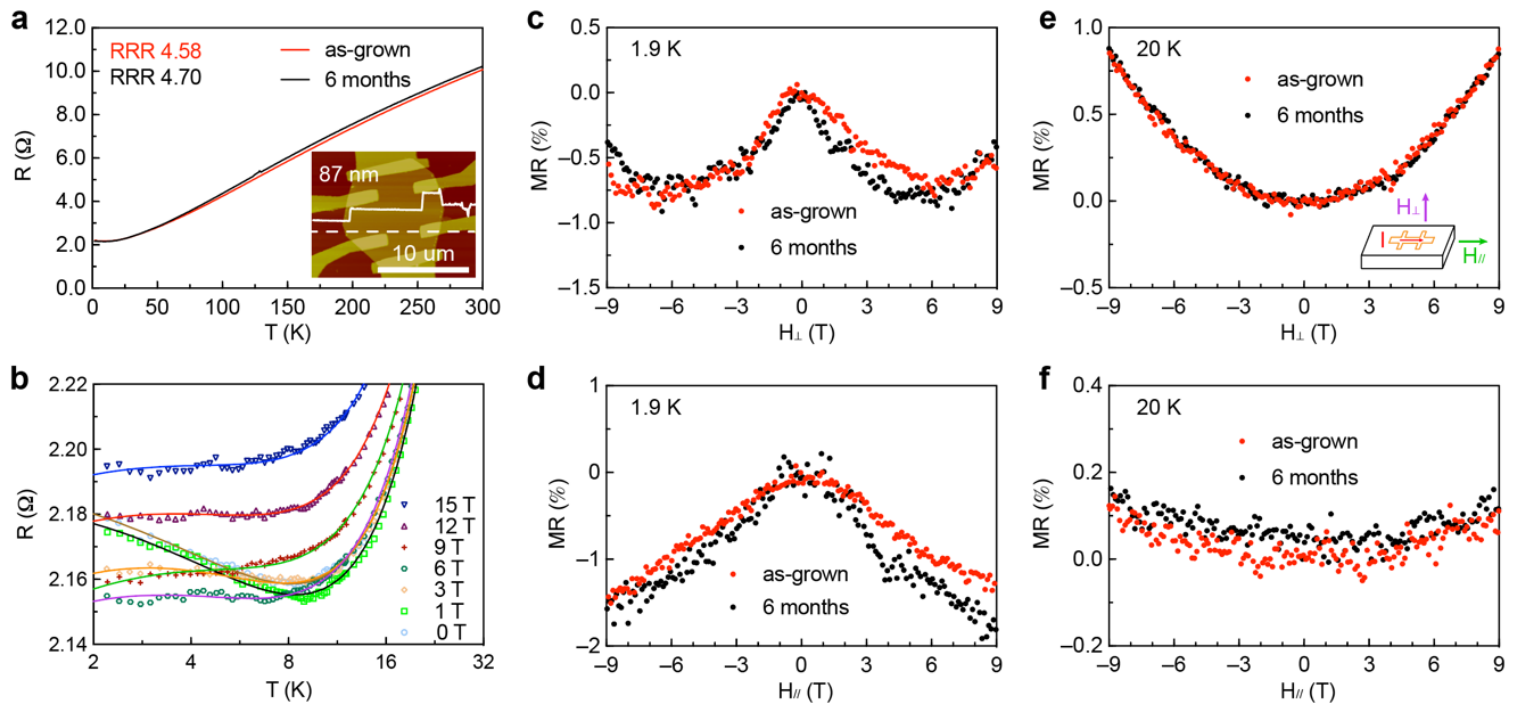

Figure S10. Comparison of device performance between the as-grown and stored half a year in a glove box filled with Ar. (a) Temperature dependent resistance of the device as-prepared and store half a year. Inset is the AFM image of the device. (b) Logarithmic low temperature dependent resistance under different magnetic field of the device stored in a glove box for half a year. (c-f) Comparison of relative $\mathrm{MR}$ at $1.9 \mathrm{~K}$ and $20 \mathrm{~K}$ with the magnetic field (c,e) perpendicular (transverse MR) and (d,f) parallel (longitudinal MR) to the plane of the sample, respectively. 

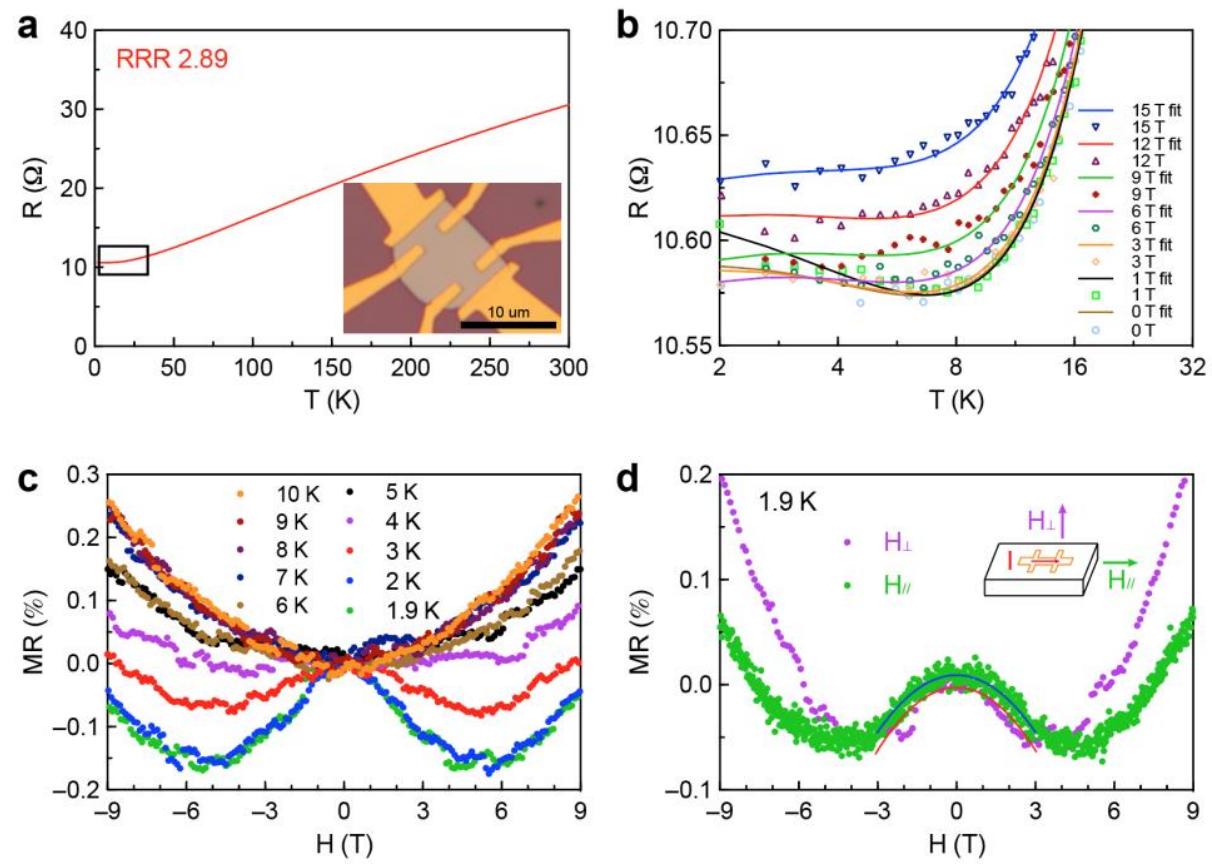

Figure S11. Low-temperature transport properties of another $\mathrm{VTe}_{2}$ nanoplate. (a) Temperature dependent resistance $(R-T)$ for the device with the optical image showed in the inset. (b) Logarithmic low temperature dependent resistance under different magnetic field (the region is highlighted by the square in (a)). (c) Relative MR at different temperatures with the magnetic field perpendicular to the plane of the sample (transverse MR). (d) Transverse and longitudinal MR with low magnetic field fits to expression $M R(\%)=a H^{2}+b$ at $1.9 \mathrm{~K}$. 

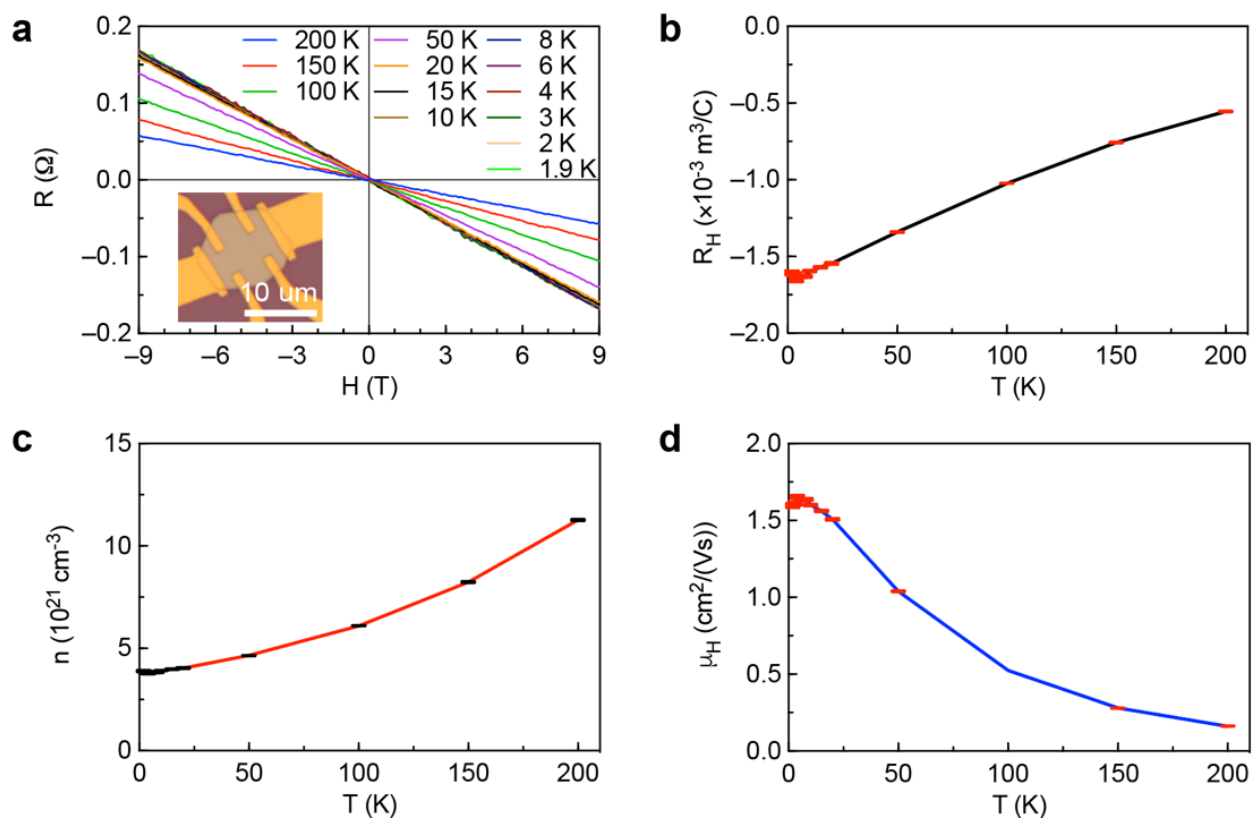

Figure S12. Variation of Hall resistance, Hall coefficient, Hall concentration and Hall mobility with temperatures. (a) Hall resistance at different temperatures. Inset is the optical image of the device. (b) Hall coefficient at different temperatures. (c) Hall concentration at different temperatures. (d) Hall mobility at different temperatures. 

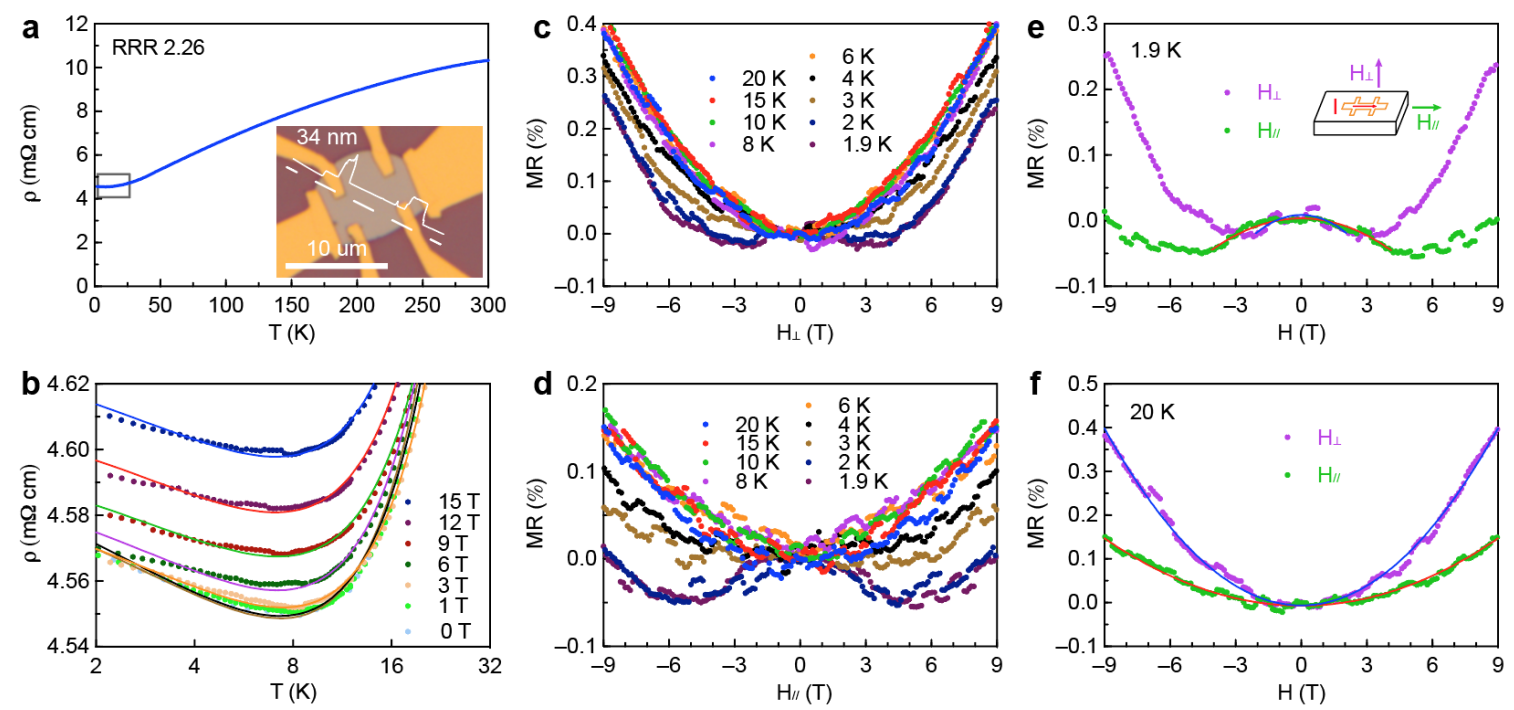

Figure S13. Low-temperature transport properties of an ultrathin $\mathrm{VTe}_{2}$ nanoplate. (a) Temperature dependent resistivity $(\rho-T)$ for the device with optical image and AFM height profile along the dash line showed in the inset. (b) Logarithmic low-temperature dependent resistivity under different magnetic field (the region is highlighted by the square in (a)). Solid lines are fits to the equation $\rho=\rho_{0}+a T^{2}-$ $b \ln T-c T^{1 / 2}$, where the first term is the residual resistivity, the second term is the Fermi liquid contribution, the third term is the Kondo resistivity, and the last term is three-dimensional electronelectron interactions. When $H \geq 3 \mathrm{~T}$, the contribution of the Kondo effect becomes negligible, indicating the suppression of the Kondo effect by strong field. (c,d) Relative MR at different temperatures with the magnetic field (c) perpendicular (transverse MR) and (d) parallel (longitudinal MR) to the plane of the sample, respectively. (e,f) Transverse and longitudinal MR with low magnetic field fits to expression $M R(\%)=a H^{2}+b$ at $1.9 \mathrm{~K}$ and $20 \mathrm{~K}$ respectively. 

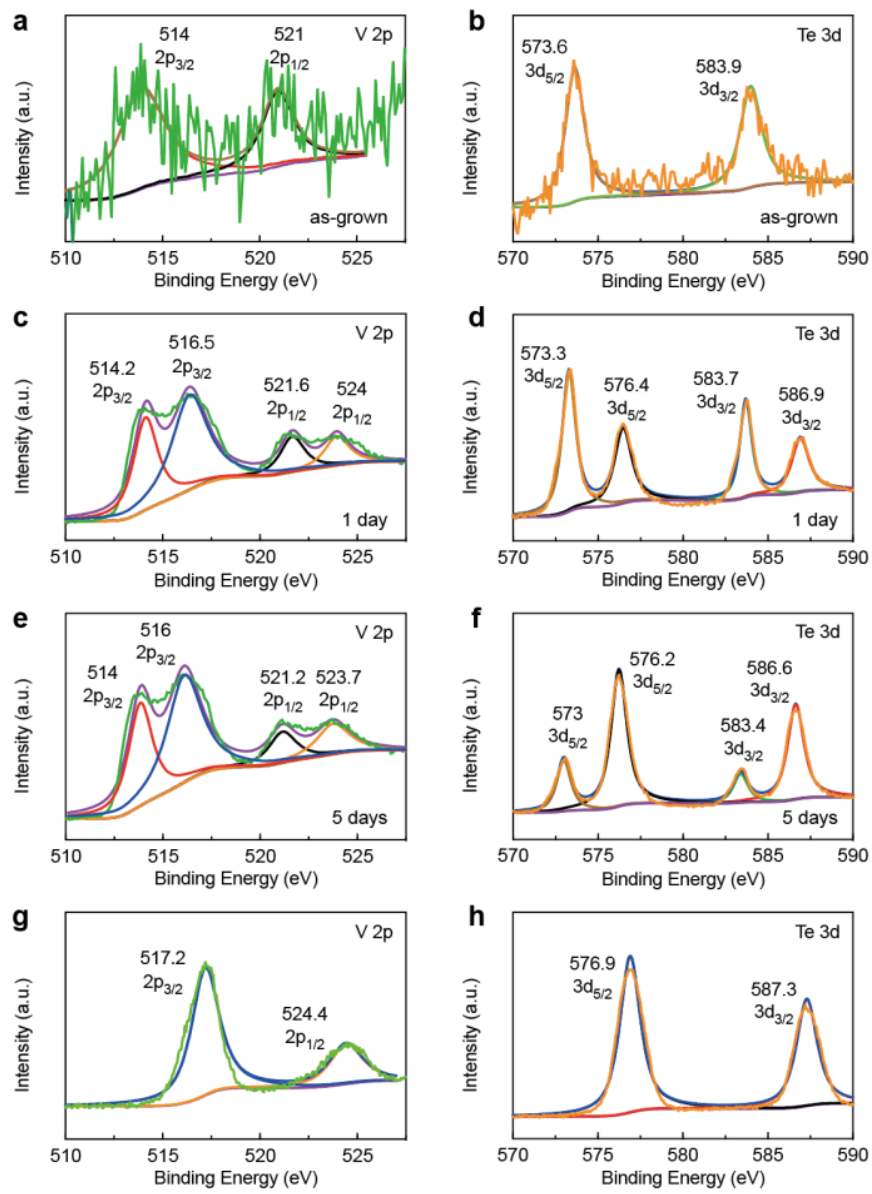

Figure S14. High resolution XPS spectra of the as-grown, degraded and completely oxidized $\mathrm{VTe}_{2}$ nanoplates. (a,b) V 2p and Te $3 d$ spectra of the as-grown $\mathrm{VTe}_{2}$ nanoplates respectively. (c,d) V 2p and Te $3 \mathrm{~d}$ spectra of the $\mathrm{VTe}_{2}$ nanoplates exposed in air for 1 day respectively. (e,f) $\mathrm{V} 2 \mathrm{p}$ and $\mathrm{Te} 3 \mathrm{~d}$ spectra of the $\mathrm{VTe}_{2}$ nanoplates exposed in air for 5 days respectively. (g,h) V $2 \mathrm{p}$ and Te $3 \mathrm{~d}$ spectra of the completely oxidized $\mathrm{VTe}_{2}$ nanoplates respectively. 
a
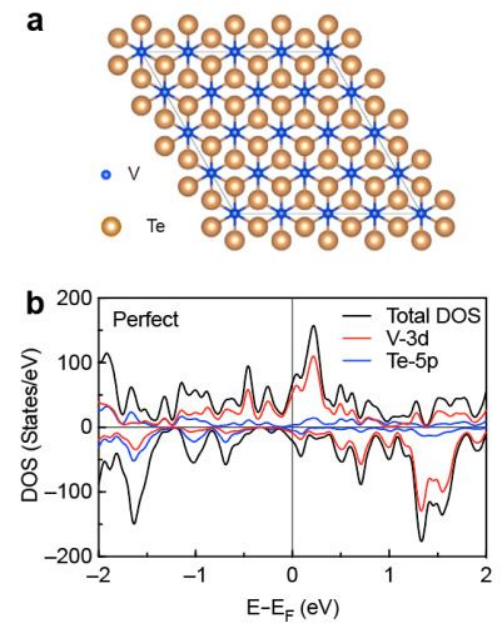

C
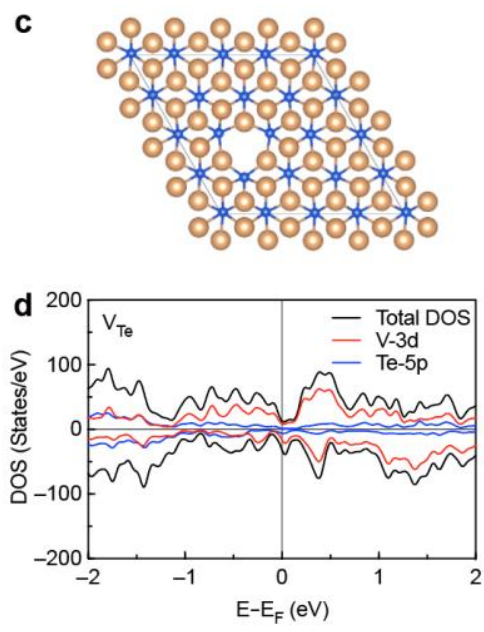

e
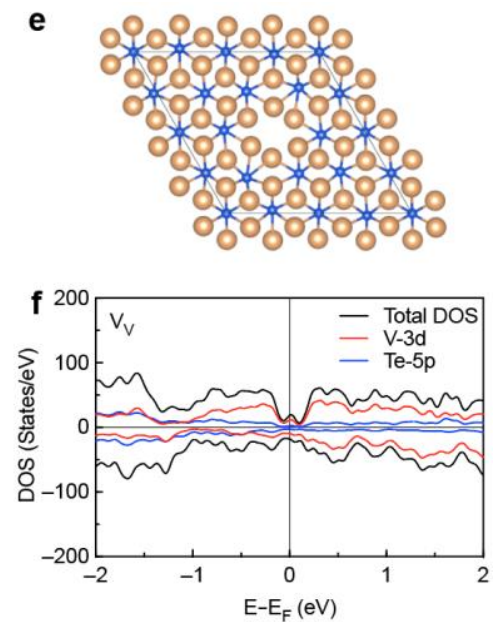

Figure S15. Structure model and DFT simulations of $\mathrm{VTe}_{2}$ with and without defects. (a) Atomic structure, and (b) total, V-3d and Te-5p projected density of states (DOS) of defect-free $\mathrm{VTe}_{2}$. (c) Atomic structure, and (d) total, V-3d and Te-5p projected DOS of $\mathrm{VTe}_{2}$ with a tellurium vacancy. (e) Atomic structure, and (f) total, V-3d and Te-5p projected DOS of $\mathrm{VTe}_{2}$ with a vanadium vacancy. The Fermi level is set to zero. Positive and negative DOS correspond to spin-up and spin-down components respectively.
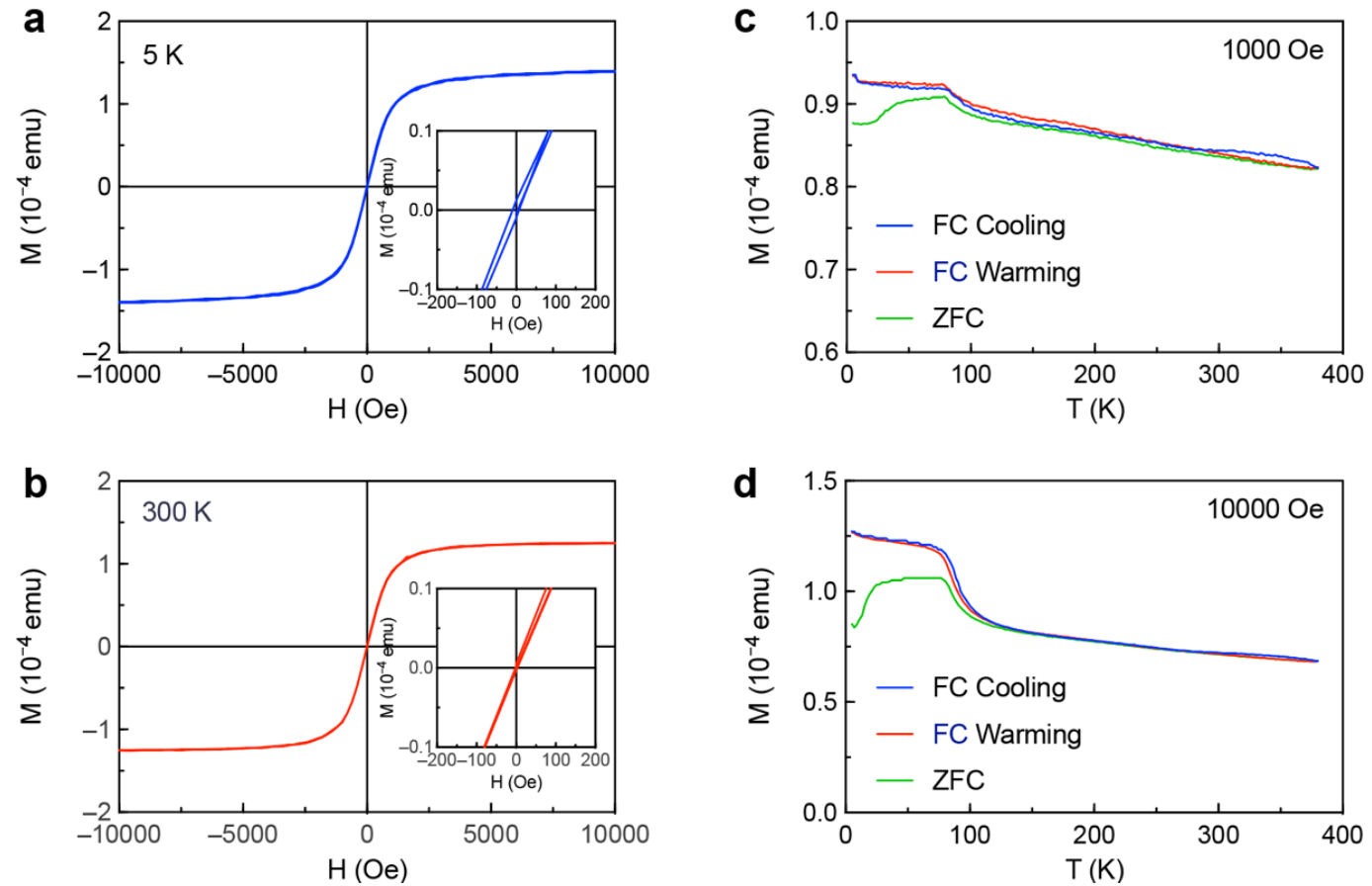

Figure S16. Magnetization measurements of the as-grown $\mathrm{VTe}_{2}$ nanoplates. (a,b) $\mathrm{M}-\mathrm{H}$ hysteresis loops taken at 5 and $300 \mathrm{~K}$ respectively. Insets are zoom-in curves of the central regions. The linear diamagnetic backgrounds from the Kapton tape are subtracted. (c,d) M-T curves measured with the external magnetic field 1000 and 10000 Oe respectively. No backgrounds are subtracted due to the weak signals of the Kapton tape. 